\title{
Más allá de los enfoques: tendencias en Psicología Clínica que trascienden las barreras teóricas. ${ }^{1}$
}

Nicolás Rodríguez Sarmiento²

\author{
nicolasrodsa@unisabana.edu.co. \\ n.rodriguez14@uniandes.edu.co
}

Artículo de reflexión recibido el 09/01/2018

y aprobado el 03/03/2018

频

Cómo citar este artículo:

Rodríguez Sarmiento, N. (2018). Más allá de los enfoques: tendencias en Psicología Clínica que trascienden las barreras teóricas. Trans-Pasando Fronteras, (11).

${ }^{1}$ Este artículo surge de una ponencia expuesta en el Tercer Encuentro Nacional de Estudiantes de Psicología (ENEPSI), Ilevado a cabo el 12 y 13 de Octubre de 2017 en las universidades ICESI y Javeriana de Cali.

2Egresado del programa de Psicología de la Universidad de La Sabana en 2017, y estudiante de la Maestría en Psicología Clínica y de La Salud de la Universidad de los Andes. 


\section{Resumen}

El ejercicio de la Psicología Clínica se encuentra en medio de la pluralidad y la diversidad, tanto de prácticas como de teorías. Este artículo presenta el marco en el que transcurre el desarrollo contemporáneo de esta disciplina, enfatizando en que las barreras de enfoque se han intentado desdibujar casi tan pronto como aparecieron. Por lo anterior, supone un desacierto formar a los estudiantes en la mutua exclusión de los enfoques teóricos (a saber: psicodinámico, conductual, cognitivo, cognitivo-conductual, humanista/existencial, sistémico, etc.). Se brinda una introducción acerca del 'problema de los enfoques' y algunas consideraciones sobre la amenaza de la pseudociencia, para luego realizar una revisión de algunas tendencias que, en el marco de la ciencia, trascienden las barreras teóricas en las que muchas veces son formados los estudiantes de psicología. Se revisan los conceptos de lo transteórico, lo transdiagnóstico, los tratamientos y la práctica basados en la evidencia (EBTs y EBP) y algunos modelos y técnicas que cumplen con estas características. También se trata brevemente la aplicación de las teorías de la complejidad y de la epistemología compleja a la psicología clínica.

Palabras clave: Integración en psicoterapia; Transdiagnóstico; Epistemología compleja; Enfoques teóricos; Revisión. 


\section{Beyond the approaches: tendencies in Clinical \\ Psychology that transcend theoretical barriers}

\section{Abstract}

The exert of Clinical Psychology can be found in the middle of plurality and diversity, both of practices and theories. This paper presents the context in which the contemporary development of this discipline elapses, emphasizing that the approach barriers have been tried to be blurred almost as soon as they appeared. This is why it is a mistake to educate the students in the mutual exclusion of theoretical approaches (namely psychodynamic, behavioral, cognitive, cognitive-behavioral, humanistic/existential, systemic, etc.). An introduction about the 'approaches problem', and certain considerations about the threat of pseudoscience are provided, to continue with a review of some tendencies that, in the frame of science, transcend the theoretical barriers in which plenty of times psychology students are trained. The concepts of transtheoretic and transdiagnostic, evidence-based treatments and practice (EBTs and EBP), and some techniques and models that meet these characteristics. The application of complexity theories and complex epistemology into Clinical Psychology are briefly discussed.

Keywords: Psychotherapy integration; Transdiagnostic; Complex epistemology; Theoretical approaches; Review. 


\section{Introducción}

Para cualquier psicólogo que pretenda una alta rigurosidad en su trabajo, es bien sabido que el abordaje del objeto de estudio de la psicología, en cualquiera de sus campos, es particularmente complejo. Al entrar en el campo de la epistemología en psicología, es posible entrever que la posmodernidad establece una crítica a los métodos de la disciplina, produciendo una pluralidad de discursos (Johnson \& Cassell, 2001). Lo anterior es transversal al enorme rango de problemáticas y aplicaciones que la psicología ha pretendido abordar históricamente, sin embargo, la psicología clínica se encuentra "en el ojo del huracán", como bien lo ilustra Opazo (1997), dada la gran heterogeneidad epistémica. Jaramillo, Escobar, y Sandoval (2015, p. 135) establecen que la posmodernidad supone para la psicología clínica "una seria confrontación con las formas tradicionales de formación, conceptualización, investigación e intervención de las problemáticas pertinentes al campo".

Bajo este contexto se desarrolla el que llamaremos "problema de los enfoques". Pese a que el presente artículo gira en torno a este tema, lo que pretende no es abordar dicho problema directamente, sino hacer un llamado a la educación y la práctica en Psicología Clínica; inicialmente en Colombia, pero puede extenderse a todo el contexto latinoamericano. Para ello se realiza una introducción al problema nombrado, para luego revisar los enfoques más aceptados y el riesgo de aceptar cualquier forma de aproximación al objeto de estudio de la psicología, junto con algunas implicaciones éticas que ello conlleva. Posteriormente se presentan algunas vertientes que se muestran como posteriores al problema de los enfoques, $y$ por último se brindan algunas consideraciones finales. 


\section{El problema de los enfoques}

En el marco descrito anteriormente se encuentra lo que -en este artículo- se entiende por el "problema de los enfoques". Ello se refiere al hecho de que en psicología (especialmente en el área clínica) existan diferentes aproximaciones epistemológicas que, en muchas ocasiones, son contrapuestas y por tanto se descalifican mutuamente. Estas aproximaciones incluyen los cinco grandes enfoques que contempla Tryon (2016b), a saber: conductual, cognitivo, cognitivo-conductual, psicodinámico, y farmacológico; pero también se deben tener en cuenta otros con igual o menor aceptación académica, como el humanista-existencial y otros relacionados (Tryon, 2016a; Winston, 2015), y el enfoque sistémico, o de sistemas (Sexton \& Stanton, 2016). Los anteriores son los de mayor reconocimiento a mi parecer, también a los ojos de Wachtel (2014; incluyendo la terapia Gestalt) y Tryon (2016a); sin embargo, el APA Handbook of Clinical Psychology incluye once capítulos, uno por cada enfoque teórico, en la primera parte de su segundo volumen, e incluso existen otros que allí no se nombran. Más allá, Brown (2015) y Paris (2013) establecen que el número de métodos clínicos está por el orden de los cientos, en parte por la comercialización y mercadeo de ciertas formas de tratamiento.

El problema descrito parece simplemente corresponder al lugar en el que se desenvuelven las Ilamadas "ciencias blandas (o suaves)", que es en medio del pluralismo y la incertidumbre. Incluso algunos sectores de la academia y la sociedad, como destaca Downing (2004, p. 124), consideran que "no es posible para la psicoterapia, o ninguna otra disciplina semejante, encontrar la verdad". Además de que esto implica un ineludible cuestionamiento sobre el alcance de la(s) teoría(s) psicológica(s), da cuenta de lo desdibujados que están los límites que encierran la parte de la psicología clínica que puede 
reconocerse como ciencia. En concreto, lo que supone la posmodernidad y la pluralidad para la psicología, es que: no solo se validan discursos no científicos, sino que queda en entredicho si la psicología es realmente una ciencia; entorno en el cual alguno se atrevería a decir que "una parte de la psicología lo es".

\section{La Pseudociencia en Psicología Clínica}

Existen diversos trabajos que intentan hacer frente a la intromisión de teorías y prácticas no científicas en el ejercicio de la psicología clínica (e. g. Lilienfeld, Lynn, \& Lohr, 2015; Hofmann, 2013). Olatunji, Parker, y Lohr (2005), por ejemplo, señalan tres formas de amenaza a lo que ellos Ilaman "Ciencia Clínica Legítima". La primera (Junk Science) es el testimonio y consejo de expertos, refiriéndose a la divulgación de discursos no calificados o faltos de soporte científico, que se difunden a través de los medios de comunicación masiva y en el contexto de procedimientos legales. La segunda amenaza (Antiscience) es tomar la verdad como un concepto ilusorio, entendiendo que la ciencia constituye solamente un modo de discurso entre muchos otros igualmente válidos, como la intuición o la experiencia personal (aspecto que se relaciona con el planteamiento descrito por Downing, 2004). La tercera (Pseudoscience) trata propiamente de la pseudociencia, indicando que la diferencia con la ciencia legítima es de grado, por lo que el concepto de pseudociencia no es categórico sino prototípico (i.e. es el polo opuesto a la ciencia dentro del mismo continuo).

El objetivo de este tipo de trabajos es el mismo que el del presente artículo: alertar a la comunidad académica (y no académica) frente a la asunción de modelos no probados por el método científico. Esto porque el propósito de superar el problema de los en- 
foques requiere, en parte, instruir al estudiante en filtrar de forma crítica e informada entre corrientes epistemológicas, para poder realizar una síntesis coherente.

Muchos de los autores mencionados toman en consideración algunos aspectos de carácter ético que van más allá del aparente cientificismo que exponen en sus trabajos. Downing (2004), por ejemplo, señala la importancia de que el psicólogo clínico emplee tratamientos que se hayan probado efectivos, a pesar de ser casi evidente. Ello implica necesariamente abandonar aquellos que hayan probado lo contrario, que es donde muchos profesionales muestran renuencia (Lilienfeld et al., 2013). Olatunji, Parker, y Lohr (2005) revisan con más detenimiento las implicaciones éticas de la pseudociencia en la práctica clínica. Una de sus consideraciones más relevantes es que la intromisión de técnicas no probadas por la ciencia da lugar a la violación del principio de no maleficencia (ver American Psychological Association, 2016), que ellos señalan como la obligación de Primum non nocere. Esto último se refiere a que usar tal tipo de técnicas abre la puerta a procedimientos potencialmente iatrogénicos (v.g. a causar daños no previstos en el paciente).

Todas estas consideraciones enmarcan el por qué se debe ser cuidadoso a la hora de aceptar cualquier teoría como válida, o a la hora de decidir militar en una sola como si se tratara de una verdad absoluta. Concretamente, no puede reducirse la práctica clínica, que procura la salud mental y la calidad de vida del consultante, al parecer del clínico. No puede ajustarse la problemática a una alternativa de solución (i.e. a un enfoque), es el problema mismo el que provee la información que se requiere para su solución, no el enfoque teórico. Este es el fin último de la psicología si ha de considerarse una ciencia. 
En este punto, vale la pena observar el estado actual de los programas de formación de pregrado y posgrado en el contexto colombiano; a fin de dar cuenta de la urgencia que supone el educar a los nuevos psicólogos clínicos en una óptica que no se limite simplemente a la de los mencionados enfoques teóricos. En este sentido, a través de una revisión de los programas de Psicología en Colombia, se puede entrever que la mayoría de los currículos pueden encasillarse en alguna de las siguientes categorías: En la primera se encuentran los que muestran al estudiante varias o todas las "grandes" aproximaciones teóricas, a la espera de que el estudiante tome una decisión informada. El problema de este modelo consiste en que no suele formar al estudiante en epistemología (i.e. en filosofía de la ciencia), sino en varias epistemologías (i.e. la de cada enfoque).

Sin herramientas suficientes para formar un criterio de filtro o de síntesis, la decisión por uno u otro enfoque se toma a partir de la mera afinidad afectiva (v.g. la que al estudiante más le guste). En la segunda categoría, se pueden ubicar los currículos que solamente toman por verdadera una corriente teórica (e. g. conductual, psicodinámica, etc.), evitando que el estudiante conozca a profundidad las demás. El problema con estos modelos es más evidente, en cuanto a que pueden obviarse contenidos relevantes de las otras corrientes; contenidos que muchas veces no son necesariamente contrarios a los que ya se imparten. Adicionalmente, como se verá más adelante, la psicología clínica tiende a un eclecticismo que no puede surgir del desconocimiento, ni de la falta de reconocimiento de la pluralidad de enfoques psicológicos, razón por la cual un profesional formado bajo esta segunda categoría de currículos no sería el más actualizado. 


\section{Más allá de los enfoques}

Este panorama parece desalentador, ya que, según está planteado, descalifica dos categorías contrarias entre sí. La solución es una tercera categoría que no se inclina por ninguna de ellas. Consiste en ir más allá de los enfoques tomando por norte el carácter científico de la psicología. En este sentido, lo que pretende la segunda parte del presente trabajo es mostrar algunas tendencias recientes de la psicología clínica que parecen trascender las antiguas barreras de los enfoques teóricos. Esta área de investigación suele enmarcarse bajo los nombres de Integración Teórica, Modelos Transteóricos, Psicoterapia Integrada, entre otros; y no es para nada nueva, ya que, como muestran Wachtel (2014), Paris (2013), y Anchin (2008), puede rastrearse hasta la década antepasada. Adicionalmente, la revista Journal of Psychotherapy Integration, una de las revistas dedicadas a esta materia, tuvo su primera aparición en 1991; momento en que se tomaba la integración psicoterapéutica como un proceso en curso (i.e. no se puede tomar el año de 1991 como un momento inicial) (ver Wachtel, 1991). Más allá de ello, Mariñelarena-Dondena (2008) reporta modelos integrativos en Latinoamérica de más de dos décadas; además de que la Asociación Latinoamericana de Psicoterapia Integrativa (ALAPSI) ubica su origen en la década de 1980. A su vez, Norcross, Goldfried, y Arigo (2016) ubican los primeros intereses de llevar a cabo una integración de la psicoterapia en la década de 1930.

Lo anterior implica, inexorablemente, que formar a un estudiante en la mutua exclusión de los enfoques teóricos (i.e. en el problema de los enfoques) consiste básicamente en brindar información desactualizada y sesgada; aun cuando el profesor no comulgue con las posturas integrativas. Por esta razón, lo que se pretende a continuación es mostrar algunas aproximaciones teóricas y 
aplicadas que parecen desdibujar las mencionadas barreras de enfoque. Esto con los propósitos, tanto de brindar actualización a quienes son y han sido formados bajo los currículos mencionados anteriormente, como de dar cuenta de la pluralidad y profundidad de los avances en lo que refiere a trascender el problema de los enfoques. La revisión planteada excluye intencionalmente yuxtaposiciones teóricas (e. g. Opazo, 1997), por tratarse de una mera sobreposición, enfatizando en la pretensión de cientificidad.

\section{La apuesta por la evidencia, y el transdiagnóstico}

Primordialmente, dada la secuencia presentada hasta ahora, se encuentra la apuesta por la evidencia empírica que proviene del intento de la medicina de los años 90 por evitar los peligros de las intervenciones que no estaban basadas en la ciencia (Katsikis, 2014). Puntualmente en psicología clínica, Kazdin (2008) ${ }^{4}$ describe los conceptos de Tratamientos Basados en la Evidencia (EBTs) y Práctica Basada en la Evidencia (EBP). El primero refiere a los métodos que "han producido cambio terapéutico en ensayos controlados" (p. 147), y difiere del segundo en que éste último lo abarca y alude a una práctica clínica experta e informada acerca de la evidencia, que incluye las necesidades y valores del consultante en la toma de decisiones a la hora de realizar una intervención psicoterapéutica (Kazdin, 2008). Este vuelco hacia la evidencia enfatiza estrictamente en la eficacia y utilidad clínica de los tratamientos (APA Presidential Task Force on Evidence-Based Practice, 2006), por encima de la conceptualización teórica de la que son provenientes, a la espera de que la evidencia dé cuenta de cuál es la aproximación más acertada para un trastorno o condición. Como se muestra más adelante, algunas de ellas son las que integran los "grandes" enfoques. A esta forma de entender la 
psicología clínica, se le ha dado el nombre de Eclecticismo Técnico (Norcross, Goldfried \& Arigo, 2016).

Si bien los EBTs y la EBP pueden entenderse como una superación del fundamentalismo teórico al que se refiere Wachtel (2014), también suponen un marco de referencia en donde se desenvuelven los avances teóricos y empíricos que buscan un indicio de verdad a través de la rigurosidad en los métodos de investigación. Estos métodos han llevado a modelos que trascienden el diagnóstico psicopatológico, de ahí que sean llamados transdiagnósticos (e. g. Kim \& Eaton, 2015). Los modelos transdiagnósticos se centran en "los procesos causales básicos que son comunes a diferentes categorías diagnósticas y que tienen una participación etiológica común en diferentes tipos de categorías observadas" (Castro, 2011, p. 46). Una de las mayores ventajas de este tipo de modelos radica en que permiten incorporar los hallazgos de diversas disciplinas y líneas de investigación al "no estar sesgado por supuestos teóricos preconcebidos” (Castro, 2011, p. 74). Lo que busca el transdiagnóstico es comprender la interacción entre procesos causales en lugar de buscar la verificación de una teoría, evitando enfrentar un posible sesgo de confirmación, que es característico de las pseudociencias (Herbert et al., 2000).

Un ejemplo prototípico de un modelo transdiagnóstico derivado en un EBT es la Terapia de Aceptación y Compromiso (ACT $)^{5}$. Este modelo incluye el Trastorno de Evitación Experiencial (TEE) como un elemento común entre diversos diagnósticos de los sistemas taxonómicos como el DSM y el CIE (Wilson \& Luciano, 2002). Además, el proceso de evitación experiencial (que en principio no es problemático, salvo cuando conduce a la inflexibilidad cognitiva), es un proceso que se reconoce en diversas orientaciones teóricas (Hayes, Strosahl, \& Wilson, 1999). Precisamen- 
te, ACT se muestra a través de la investigación como "eficaz en un amplio rango de problemas en los que un patrón común de evitación experiencial [...] está presente" (Ruiz, 2010, p. 146). A su vez, la validación empírica del tratamiento permite sospechar la certeza de conceptualizaciones teóricas como la Teoría de Marcos Relacionales, la Fusión y Defusión Cognitiva, Aceptación, Mindfulness, entre otros (cf. Hayes, 2016); y de técnicas como el uso de metáforas (Stoddard \& Afari, 2014). Es de esta manera como se da un giro en el que los modelos terapéuticos, que originalmente se desarrollan en función de las categorías diagnósticas, pasan a ser una fuente de información para los sistemas de clasificación (Hofmann, 2013).

En general, los modelos terapéuticos basados en las teorías de aceptación y mindfulness muestran una alta eficacia, en parte porque surgen en medio del giro hacia los tratamientos basados en la evidencia (Follette \& Hazlett-Stevens, 2016). Entre ellos se encuentran la Terapia Dialéctico-Conductual (DBT; Linehan, 1993, 2015), que se conceptualiza también como un tratamiento de uso transdiagnóstico (Ritschel, Lim, \& Stewart, 2015); por supuesto la Terapia de Aceptación y Compromiso (ACT); y los tratamientos basados en mindfulness, como el MBSR (Mindfulness Based Stress Reduction) (Kabat-Zinn, 2003). Uno de los aspectos interesantes de la práctica de mindfulness es su origen: proviene del budismo y fue introducida al mundo clínico en occidente por un PhD en biología molecular (Jon Kabat-Zinn), teniendo un crecimiento exponencial como un área de investigación y encontrando un soporte empírico significativo (Follette \& Hazlett-Stevens,

${ }^{4}$ La APA ofrece un índice de tratamientos eficaces para diferentes trastornos en el sitio web de su doceava división: https://www.div12.org/psychological-treatments/

${ }^{5}$ Para una revisión rápida de las tres generaciones de terapia cognitivo-conductual, y la historia y conceptualización de la Terapia de Aceptación y Compromiso, ver Hayes, 2016. 
2016; Shapiro, 2009). La relevancia de la historia del mindfulness radica en que las técnicas contempladas por la EBP pueden tener proveniencias inesperadas. Por ello hay que moverse con cautela en el terreno de la innovación en psicología clínica, dado que existe el riesgo tanto de caer en la pseudociencia, como de realizar descubrimientos determinantes para el desarrollo de la disciplina.

\section{Modelos transteóricos e integración teórica}

La práctica de mindfulness es un ejemplo transversal a la mayoría de los temas abordados en este artículo, probablemente porque es un concepto muy amplio y flexible. Dentro y fuera de la psicología, "abundan las definiciones de 'mindfulness'”(Simón, 2010, p. 163) pese a que es un constructo relativamente simple que alude a una práctica o actitud de consciencia plena; por supuesto, con una profunda y robusta conceptualización (cf. Siegel, 2011). En este sentido, mindfulness se presenta como un constructo transteórico, por ser transversal entre las psicoterapias efectivas (bajo nombres diferentes) y al ser utilizado por terapeutas de diferentes enfoques teóricos, entre ellos el cognitivo-conductual, el psicodinámico, y el humanista (Siegel, Germer, \& Olendzki, 2008).

El anterior fue un ejemplo introductorio sobre los planteamientos que atraviesan los diferentes enfoques teóricos, en este caso, sin pretenderlo. Sin embargo, existen trabajos que abordan directamente el problema de los enfoques, como los de Wachtel (1982, 1991, 2014, 2017), quien se centra en los puntos comunes y puentes entre las posturas psicodinámicas y la terapia de conducta. Tryon (2016b) establece, sobre el trabajo de Wachtel, que tuvo éxito más en el campo de la aplicación que en el de la integración teórica. A su vez lo que éste último busca a través de 
su trabajo investigativo es establecer una adecuada integración de la psicoterapia a través de una unificación teórica mediada por las neurociencias (Tryon, 2014, 2016a, 2016b).

Esto se lleva a cabo a través de modelos y simulaciones de redes neuronales en los que se buscan principios relacionados con los postulados de las 'cinco grandes orientaciones clínicas' (i. e. conductual, cognitiva, cognitivo-conductual, psicodinámica, y farmacológica) (Tryon, 2016b) a las que posteriormente añade una sexta: Experiencial/Humanista/Existencial (Tryon, 2016a).

Esta empresa no es del todo novedosa, y supone una sólida y extensa línea de trabajo en diversas disciplinas (psicología cognitiva, ciencias computacionales, neurología clínica, neurociencia, neurofilosofía, etc.) que traza un puente entre las ciencias naturales y las ciencias humanas, bajo el nombre de Neurociencia Cognitiva (Ilardi \& Feldman, 2001). La apuesta de Warren Tryon (y otras afines) es tremendamente ambiciosa, en el sentido en que busca una integración teórica de los enfoques al buscar identificar los mecanismos causales que inevitablemente son transversales a ellos, a través de la neurociencia. Esto, de no ser claro hasta ahora, consiste en incluir a la psicología dentro del marco de las ciencias naturales (Tryon, 2014); lo que por supuesto tiene enormes implicaciones que no se pretenden abordar en éste artículo, pero incluyen (en mi opinión) la propuesta más cientificista (sin la intención de usar la acepción peyorativa del término) de la psicología clínica hasta ahora.

No obstante, por las limitaciones de los métodos de que disponemos para tal forma de investigación, siguen siendo requeridas las consideraciones puramente teóricas (aunque deriven de métodos fisicalistas), por lo que Tryon (2016a) toma en considera- 
ción los conceptos nucleares críticos (critical core concepts) de cada enfoque para la unificación teórica. La última propuesta podría entenderse como un intento que parte de la observación de la naturaleza para la (re)construcción del paradigma epistemológico de la psicología; sin embargo, existe un punto de vista opuesto que pretende ajustar un nuevo paradigma a las demandas del mundo contemporáneo, lo que tiene implicaciones a posteriori sobre los métodos de investigación (i.e. sobre la observación de la naturaleza).

Este es el caso de la aplicación de las teorías de la complejidad a la Psicología Clínica (Jaramillo, 2009; Jaramillo et al., 2015). Morin (1996) entiende a la complejidad como un giro de la simplificación y reducción de las ciencias tradicionales (v.g. del método analítico) hacia una comprensión integradora del mundo que lo entiende en su totalidad como una relación entre orden, desorden y organización. Se trata de una concepción transdisciplinar que figura como un (nuevo) paradigma epistemológico, y surge de la insuficiencia del paradigma hegemónico de la ciencia para abordar los problemas contemporáneos (Maldonado, 2009; Munné, 2005).

Como se puede observar en el estado del arte de Maldonado y Gómez (2010), son muchas disciplinas las que se pueden categorizar dentro de las ciencias de la complejidad, y a pesar de que en psicología solo reconocen la aplicación de la teoría de sistemas, el paradigma de la complejidad tiene mayores implicaciones para el área clínica. Estas implicaciones son descritas ampliamente por Jaramillo (2009), quien ensambla un método clínico fundamentado en la 'epistemología compleja'. Entre ellas destaca el hecho de que, bajo este paradigma, los puntos de vista opuestos no son mutuamente excluyentes. Ello conlleva la posibilidad de construir modelos integrativos a partir del conjunto de teorías existentes. 


\section{Conclusiones}

Ante la pluralidad de teorías y prácticas a las que está sujeta la Psicología (especialmente la clínica), existe una necesidad de establecer un filtro y encaminar los esfuerzos en direcciones afines y complementarias. Pese a que la diversidad es una fuente para hallazgos importantes, como en el caso de la práctica de mindfulness, y la ciencia se nutre del error y del accidente (Skinner, 1971); existe una razón de peso para depurar la práctica clínica de formas las de intervención no-científicas.

Esta razón es de tipo ético, busca proteger al cliente de una posible iatrogenia o de intervenciones que no funcionan, y se enuncia constantemente en el ámbito académico (e.g. American Psychological Association, 2016; APA Presidential Task Force on Evidence-Based Practice, 2006; Brown, 2015; Herbert et al., 2000; Lilienfeld, Lynn, \& Lohr, 2015; Lilienfeld, Ritschel, Lynn, Cautin, \& Latzman, 2013; Olatunji, Parker, \& Lohr, 2005).

En este contexto se desenvuelve el nombrado problema de los enfoques, que consiste, según lo visto, en una mutua desacreditación entre perspectivas teóricas que ha sido trabajada (y muchas veces superada) ampliamente. Por lo mismo resulta impertinente que se siga formando a los estudiantes de pregrado y posgrado en la mutua exclusión de los enfoques teóricos. Ello implica la promoción de una disciplina desunificada en donde unos profesionales desacreditan a otros, y comprometen la integridad e imagen de la psicología. Adicionalmente, los programas que conciben la formación en teorías mutuamente excluyentes suelen promover (probablemente de forma inintencionada) la desinformación acerca de los esfuerzos por la integración. Por esta razón, se presentaron diversas posturas que trascienden el problema de los enfoques. 
La que parece más relevante es la apuesta por la evidencia, ya que deja en un segundo lugar los debates teóricos frente a la eficacia y efectividad de las intervenciones. El método más empleado y confiable para brindar evidencia sobre los modelos terapéuticos es el ensayo aleatorizado (Randomized Controlled Trial: RCT; Katsikis, 2014). No obstante, en palabras de John C. Norcross, es insuficiente por no tener en cuenta el carácter interpersonal (por tanto ideográfico) del proceso terapéutico (Wolf, 2003). En general, los métodos con los que cuenta la psicología clínica para acercarse a su objeto de estudio siguen siendo imprecisos. Al respecto, Brown (2015) ha escrito que, frente a la diversidad de modelos terapéuticos y la insuficiencia de los instrumentos para medir su efectividad, es importante concentrarse en los aspectos comunes más que en las diferencias. También Chorpita, Miranda, y Bernstein (2011) enfatizan en la necesidad de una "arquitectura común", refiriéndose a que se tengan estándares comunes en la práctica clínica para facilitar un crecimiento unificado en la investigación. Por esta razón se muestran los modelos trans- e interteóricos, así como los transdiagnósticos, como una salida al problema de los enfoques; modelos que aseguran su pertinencia al estar empíricamente validados como EBTs.

Cuando un psicólogo clínico se declara a sí mismo especialista en alguno de los enfoques (i.e. conductista, psicoanalista, humanista, etc.), desconoce necesariamente la utilidad y pertinencia de los demás. Ser especialista dentro de una profesión se refiere al área de aplicación en la que una persona es experta (e.g. niños y adolescentes, trastornos de ansiedad, consumo de SPA, etc.), no a un cuerpo teórico particular. Lo concluyente de este artículo, es que las barreras de enfoque sientan sus bases en el desconocimiento y la mutua descalificación, y limitan el alcance y el desarrollo de la disciplina. A pesar de que existe una tendencia o línea de trabajo 
reciente hacia la integración en psicoterapia, que la ha llevado a reconocerse como un movimiento (Boswell, 2017), no se trata de un tema nuevo, como se observa en Norcross, Goldfried, y Arigo (2016). Como se ha mostrado, los instrumentos con los que ahora cuenta Neurociencia Cognitiva permiten un acercamiento más serio a la integración teórica, lo que se evidencia en el trabajo de Warren Tryon (2014, 2016a, 2016b). Bajo este panorama, existe una expresa invitación para los psicólogos que atraviesan su periodo de formación, y para los formadores, a que no limiten su conocimiento ni su ejercicio a las antiguas barreras teóricas.

\section{Referencias $^{6}$}

American Psychological Association (2016). Ethical Principles of Psychologists and Code of Conduct. In A. E. Kazdin (Ed.), Methodological issues and strategies in clinical research (pp. 495512). http://doi.org/10.1037/0003-066X.57.12.1060

Anchin, J. C. (2008). Pursuing a unifying paradigm for psychotherapy: Tasks, dialectical considerations, and biopsychosocial systems metatheory. Journal of Psychotherapy Integration, 18(3), 310-349. http://doi.org/10.1037/a0013557

APA Presidential Task Force on Evidence-Based Practice. (2006). Evidence-based practice in psychology. American Psychologist, 61, 271-285. http://doi.org/10.1002/jclp.20384

¿La riqueza de este artículo se encuentra en las referencias consultadas, incluso más que en el contenido mismo. Se recomienda enfáticamente que los interesados den una detenida revisión a la bibliografía. 
Boswell, J. F. (2017). Psychotherapy Integration: Research, Practice, and Training at the Leading Edge. Journal of Psychotherapy Integration, 27(2), 225-235. http://doi.org/10.1037/int0000055

Brown, J. (2015). Specific techniques vs. Common factors? Psychotherapy integration and its role in ethical practice. American Journal of Psychotherapy, 69(3), 301-316.

Castro, L. (2011). Modelo integrador en psicopatología: un enfoque transdiagnóstico. In V. E. Caballo, I. C. Salazar, \& J. A. Carrobles (Eds.), Manual de psicopatología y trastornos psicológicos. Madrid: Ediciones Pirámide.

Chorpita, B. F., Miranda, J., \& Bernstein, A. (2011). Creating Public Health Policy: The Dissemination of Evidenced Based Psychological Interventions. In D. H. Barlow (Ed.), The Oxford Handbook of Clinical Psychology (pp. 210-220). New York: Oxford University Press.

Downing, J. N. (2004). Psychotherapy practice in a pluralistic world: Philosophical and moral dilemmas. Journal of Psychotherapy Integration, 14(2), 123-148. http://doi.org/10.1037/1053-0479.14.2.123

Follette, V. M., \& Hazlett-Stevens, H. (2016). Mindfulness and acceptance theories. In J. C. Norcross, G. R. VandenBos, \& D. K. Freedheim (Eds.), APA handbook of Clinical psychology: Theory and research (Vol. 2, pp. 273-302). American Psychological Association. http://doi.org/10.1037/14773-010

Hayes, S. C. (2016). Acceptance and Commitment Therapy, Relational Frame Theory, and the Third Wave of Behavioral and Cognitive Therapies - Republished Article. Behavior Therapy, 47(6), 869-885. http://doi.org/10.1016/j.beth.2016.11.006 
Hayes, S. C., Strosahl, K. D., \& Wilson, K. G. (1999). Acceptance and commitment therapy: An experiential approach to behavior change. New York: The Guilford Press.

Herbert, J. D., Lilienfeld, S. O., Lohr, J. M., Montgomery, R. W., O'Donohue, W. T., Rosen, G. M., \& Tolin, D. F. (2000). Science and pseudoscience in the development of eye movement desensitization and reprocessing: Implications for clinical psychology. Clinical Psychology Review, 20(8), 945-971. http://doi.org/10.1016/ S0272-7358(99)00017-3

Hofmann, S. G. (2013). Bridging the theory-practice gap by getting even bolder with the boulder model. Behavior Therapy, 44(4), 603-608. http://doi.org/10.1016/j.beth.2013.04.006

Ilardi, S. S., \& Feldman, D. (2001). The Cognitive Neuroscience Paradigm: A Unifying Metatheoretical Framework for the Science and Practice of Clinical Psychology. Journal of Clinical Psychology, 57(9), 1067-1088.

Jaramillo, J. C. (2009). Consideraciones identitarias para una psicología fundada en la epistemología compleja. International Journal of Psychological Research, 2(2), 158-166.

Jaramillo, J. C., Escobar, A., \& Sandoval, C. (2015). Aproximaciones a una clínica psicológica y su método, fundamentada en la epistemología compleja y adecuada a contextos postmodernos. Revista CES Psicología, 8(1), 134-154.

Johnson, P., \& Cassell, C. (2001). Epistemology and work psychology:newagendas. Journal of Occupational and Organizational Psychology, 74(2), 125-143. http://doi.org/10.1348/096317901167280 
Kabat-Zinn, J. (2003). Mindfulness-based stress reduction (MBSR). Constructivism in the Human Sciences, 8(2), 73-107. Retrieved from https://goo.gl/dv4uHW

Katsikis, D. (2014). Evidence-based research: the importance for the present and future of evidence-based practice. Journal of Evidence-Based Psychotherapies, 14(2), 259-270. Retrieved from https://goo.gl/aZyibv

Kazdin, A. E. (2008). Evidence-based treatment and practice: New opportunities to bridge clinical research and practice, enhance the knowledge base, and improve patient care. American Psychologist, 63(3), 146-159. http://doi.org/10.1037/0003-066X.63.3.146

Kim, H., \& Eaton, N. R. (2015). The hierarchical structure of common mental disorders: Connecting multiple levels of Comorbidity, bifactor models, and predictive validity. Journal of Abnormal Psychology, 124(4), 1064-1078. http://doi.org/10.1037/ abn0000113

Lilienfeld, S. O., Lynn, S. J., \& Lohr, J. M. (2015). Science and Pseudoscience in Clinical Psychology. New York: The Guilford Press.

Lilienfeld, S. O., Ritschel, L. A., Lynn, S. J., Cautin, R. L., \& Latzman, R. D. (2013). Why many clinical psychologists are resistant to evidence-based practice: Root causes and constructive remedies. Clinical Psychology Review, 33(7), 883-900. http://doi. org/10.1016/j.cpr.2012.09.008

Linehan, M. M. (1993). Cognitive-Behavioral Treatment of Borderline Personality Disorder. New York: The Guilford Press. 
Linehan, M. M. (2015). DBT Skills Training Manual (Second Edition). New York: The Guilford Press. http://doi.org/10.1093/alcalc/agv004

Maldonado, C. E. (2009). La complejidad es un problema, no una cosmovisión. UCM Revista de Investigación, (13), 42-54.

Maldonado, C. E., \& Gómez, N. A. (2010). El mundo de las ciencias de la complejidad. Un estado del arte. Bogotá: Editorial Universidad del Rosario.

Mariñelarena-Dondena, L. (2008). Psicología positiva y modelos integrativos en psicoterapia. Fundamentos En Humanidades, 9(28), 55-69.

Morin, E. (1996). A New Way of Thinking. The Unesco Courier. Secrets of Complexity, 2, 10-14.

Munné, F. (2005). ¿Qué es la complejidad? Encuentros de Psicología Social, 3(2), 6-17.

Norcross, J. C., Goldfried, M. R., \& Arigo, D. (2016). Integrative Theories. In J. C. Norcross, G. R. VandenBos, \& D. K. Freedheim (Eds.), APA Handbook of Clinical Psychology: Theory and Research (Vol. 2, pp. 303-332). American Psychological Association.

Olatunji, B. O., Parker, L. M., \& Lohr, J. M. (2005). Pseudoscience in contemporary psychology: Professional issues and implications. The Scientific Review of Mental Health Practice, 4(2), 19-36. Retrieved from http://search.ebscohost. $\mathrm{com} /$ ogin. aspx?direct $=$ true $\& d b=$ psyh $\& A N=2008-00021$ 006\&site=ehost-live\%5Cnoolatun@mail.uark.edu 
Opazo, R. (1997). In the Hurricane's Eye: A Supraparadigmatic Integrative Model. Journal of Psychotherapy Integration, 7(1), 1754. http://doi.org/10.1037/h0101136

Paris, J. (2013). How the history of psychotherapy interferes with integration. Journal of Psychotherapy Integration, 23(2), 99106. http://doi.org/10.1037/a0031419

Ritschel, L. A., Lim, N. E., \& Stewart, L. M. (2015). Transdiagnostic applications of DBT for adolescents and adults. American Journal of Psychotherapy, 69(2), 111-128. http://doi.org/http://dx.doi. org/10.1108/17506200710779521

Ruiz, F. (2010). A review of acceptance and commitment therapy (ACT) empirical evidence: Correlation, experimental, psychopathology, component and outcome studies. International Journal of Psychology and Psychological Therapy, 10, 125-162. http://doi. org/10.1080/028457199439937

Sexton, T. L., \& Stanton, M. (2016). Systems Theories. In J. C. Norcross, G. R. VandenBos, \& D. K. Freedheim (Eds.), APA Handbook of Clinical Psychology: Theory and Research (Vol. 2, pp. 213-239). American Psychological Association. http://doi. org/10.4135/9781412958479

Shapiro, S. L. (2009). The Integration of Mindfulness and Psychology. Journal of Clinical Psychology, 65(6), 555-560. http:// doi.org/10.1002/jclp

Siegel, R. D. (2011). La Solución Mindfulness. Prácticas cotidianas para problemas cotidianos. (B. Moreno, Ed.). Bilbao: Desclée De Brouwer, S. A. 
Siegel, R. D., Germer, C. K., \& Olendzki, A. (2008). Mindfulness: What is it? Where Does It Come From? In F. Didonna (Ed.), Clinical Handbook of Mindfulness (pp. 17-35). New York: Springer. http:// doi.org/10.1080/14639947.2011.564813

Simón, V. (2010). Mindfulness y psicología: presente y futuro. Informació Psicològica, 100, 162-170. Retrieved from http://dialnet. unirioja.es/descarga/articulo/3642934.pdf\%5Cnhttp://dialnet. unirioja.es/servlet/extart?codigo $=3642934$

Skinner, B. F. (1971). Beyond Freedom \& Dignity. Indianapolis: Hackett Publishing Company.

Stoddard, J. A., \& Afari, N. (2014). The Big Book of ACT Metaphors. A practitioner's Guide to Experiential Exercises \& Metaphors in Acceptance \& Commitment Therapy. Oakland: New Harbinger Publications.

Tryon, W. W. (2014). Cognitive neuroscience and psychotherapy: Network principles for a unified theory. New York: Academic Press.

Tryon, W. W. (2016a). Psychotherapy Integration via Theoretical Unification. International Journal of Integrative Psychotherapy, 7 , 1-26. Retrieved from http://integrative-journal.com/index.php/ ijip/article/view/106

Tryon, W. W. (2016b). Transtheoretic transdiagnostic psychotherapy. Journal of Psychotherapy Integration, 26(3), 273287. http://doi.org/10.1037/a0040041

Wachtel, P. L. (1982). What can dynamic therapies contribute to behavior therapy? Behavior Therapy, 13(5), 594-609. 
Wachtel, P. L. (1991). From electicism to synthesis toward a more seamless psychotherapeutic integration. Journal of Psychotherapy Integration, 1(1), 43-54. http://doi.org/10530479/91/0300-0043506.500

Wachtel, P. L. (2014). On the limits of theoretical fundamentalism. Journal of Psychotherapy Integration, 24(2), 95-98. http://doi. org/10.1037/a0036973

Wachtel, P. L. (2017). Pathways to Progress for Integrative Psychotherapy: Perspectives on Practice and Research. Journal of Psychotherapy Integration. Advance online publication. http:// dx.doi.org/10.1037/int0000089

Wilson, K. G., \& Luciano, M. C. (2002). Terapia de Aceptación y Compromiso (ACT). Un tratamiento conductual orientado a los valores. Madrid: Ediciones Pirámide.

Winston, C. N. (2015). Points of Convergence and Divergence Between Existential and Humanistic Psychology: A Few Observations. Humanistic Psychologist, 43(1), 40-53. http://doi.org/10.1080/08 873267.2014.993067

Wolf, A. W. (2003). Pragmatism, Pluralism, and Psychotherapy Relationships: An Interview with John C. Norcross, Ph.D. Journal of Contemporary Psychotherapy, 33(2), 129-143. http://doi. org/10.1023/A:1022887205178. 